\title{
Perspectivas actuales en Psicología de la Religión
}

\author{
Jorge Garcés
}

Universidad de Valencia

\section{INTRODUCCION}

Cuando revisamos la bibliografia referente al tema "Actitudes religiosas", nos damos cuenta de que las investigaciones al respecto son muy escasas. Desde hace algunos años, los psicólogos sociales han descuidado la problemática psicosocial de la religión de tal modo que no sólo no se llevan a cabo trabajos, como aludiamos más arriba, sino que los estudios realizados están altamente saturados por una fuerte carga ideológica, desembocando el contenido de las investigaciones en una suerte de partidismo. De perdurar mucho este tono polémico, el avance científico en el campo de la Psicología de la Religión se verá coartado por la pugna de sus mismos creadores.

Pero, ¿qué se entiende por Psicología de la Religión?. Poveda (1967), señalaba que la Psicología de la Religión, o se ocupa de la religión en su aspecto de fenómeno psíquico o zozobra, como así ha ocurrido, en su intento de convertirse en una descripción plenamente significativa de algo como es la "religiosidad», que no es sino un capítulo de "la historia del alma humana». Por su parte, Stollberg (1979) arguye que la Psicología de la Religión es el estudio de las constantes psicológicas de la religiosidad, y que, por tanto, no indaga sobre la verdad divina, pues ello concierne a la teología, sino sobre la realidad humana en la que aparece la fe en una revelación divina. Las tareas y objetivos que abarca la Psicología de la Religión según el citado autor son, ante todo, el profundizar y analizar criticamente las realidades religiosas; es decir, el carácter proyectivo e ideal de las religiones, la 
función racionalizadora de las teologías, el sentido de los tabúes y los prejuicios, así como los mecanismos de defensa que se manifiestan de diversas formas en las religiones para suprimir la angustia.

Si analizamos con detalle las posiciones anteriores, nos percataremos de que su contenido es bien distinto, lo que a su vez nos da idea de las significativas diferencias de criterio que existen entre los estudiosos del tema. La clarificación de tales problemas analíticos se vuelve aún más incierta ante la ya mencionada escasez de trabajos rigurosos (Michaels, 1956). Este autor hizo un rastreo de los trabajos sobre religión publicados en Psychological Abstracts en el segundo cuarto de siglo (1927-1953) y halló que únicamente el 12 por 100 eran estudios de naturaleza netamente empírica, con un promedio menor a seis por año. Más tarde, McDowell (1963) revisó la «Bibliografia comentada en Psicología y Religión” de Meissner (1961) encontrando que el volumen de publicaciones en revistas tales como «Psicología Pastoral» dentro de la década de 1950-60, no era empírico ni experimental, y en la mayoría de los casos se reducía a someras reseñas de estudios de un solo caso.

Lo paradójico del caso proviene de la incidencia de lo «religioso» en la vida social; Lenski (1961) señala en sus estudios cómo las organizaciones religiosas no sólo siguen siendo influyentes en la sociedad actual, sino que su impacto alcanza a todos los sistemas institucionales de la comunidad en las que esos individuos participan. Desde otra perspectiva, pero subrayando la importancia del hecho, Cline y Richards (1965) apuntaron que en función del grupo socio-religioso al que una persona pertenece, aumentarán o disminuirán las probabilidades de que se vote o no a determinados partidos y organizaciones políticas, la familia sea numerosa, se esté a favor o en contra de la integración racial, etc.

Esta escasez de estudios al respecto es atribuida por Strunk (1958) al rápido crecimiento y proliferación del conductismo, que abogó desde su nacimiento por un abandono de todo lo que comportaba conducta compleja y, en consecuencia, conducta religiosa. Por lo que a temas concretos se refiere, se ha incidido especialmente sobre la explicación de actos rituales, las experiencias místicas y extraordinarias, las vivencias de conversión, representaciones de la fantasia, etc. No obstante y desde hace algunos años se tiende a uparticularizar las cuestiones, a la vez que se amplían los enfoques y unidades de análisis", como afirma Tornos (1979). El interés de los investigadores se decanta hoy hacia temas tales como afiliación religiosa y salud mental, simbólica del inconsciente y religión, personalidad autoritaria y práctica religiosa, interpretación de la fe, etc.

\section{LA POLEMICA ENTRE PSICOLOGIA Y TEOLOGIA}

En la actualidad, uno de los problemas a resolver dentro del campo de la Psicosociología de la Religión viene expresado por los deba- 
tes entablados entre psicólogos y teólogos acerca de la legitimidad de unos u otros para investigar sobre este particular.

Según Mankeliunas (1961) son tres las posiciones al respecto:

1. Los empiristás sostienen que nadie mejor que los desprovistos de religiosidad o de todo sentimiento religioso, para estudiar imparcialmente el hecho religioso, ya que una determinada creencia orientaría de antemano la investigación psicológica.

2. ${ }^{2} \quad$ La posición contraria sostiene que únicamente debe estudiar la Psicología de la Religión «el psicólogo religioso», pues el hecho religioso sólo puede llegar a conocerlo quien subjetivamente lo experimenta.

3. $\quad$ La postura intermedia intenta buscar el equilibrio entre las dos partes anteriores, de tal forma que el mejor modo para el estudio del hecho religioso es la de aquellos que habiendo profesado una religión, la abandonan después.

Estas opciones se mantienen aún en nuestros días, aunque se tiende paulatinamente a radicalizar la cuestión entre los empiristas y los que se hacen llamar "psicólogos religiosos». Entre estos últimos se encuentra el propio Mankeliunas, para quien sólo el psicólogo con convicciones religiosas puede penetrar en el misterio de la religiosidad. Francis (1978) sugiere que las investigaciones psicológicas de la religión y los fenómenos con ella relacionados están acosados por problemas formidables como el que estamos tratando. Admite que los teólogos malinterpretan en ocasiones lo que los psicólogos de la religión intentan hacer, pero, al mismo tiempo, enfatiza que el error más grande de esos psicólogos es el de «meterse a hacer religión» cuando no son competentes para ello; tal incompetencia vendria dada por el hecho de que a menudo el psicólogo no se ha preocupado por la teología filosófica, dejando así de considerar los peculiares problemas epistemológicos que comporta tanto la creencia religiosa como la genuina conducta psicolingüística de los teólogos; esto es así, sigue diciendo Francis, porque el juego del lenguaje religioso sólo puede ser entendido por el sujeto que está inserto en esa «forma de vida religiosa».

Aunque las aportaciones de Francis ponen de relieve que únicamente los psicólogos religiosos pueden llevar a cabo estudios sobre religión, dejando de lado aquellas investigaciones de corte empirista, moderará posteriormente sus críticas y abogará por un marco multidisciplinar para que los trabajos sobre Psicología de la Religión puedan avanzar de manera significativa.

Frente a las tesis defendidas por Francis y Mankeliunas, cabe destacar la formulada por Harris (1978), señalando que si en verdad los teólogos desean defender el status del lenguaje religioso, estará también en ellos la explicación a los escépticos y ateos de la significación de sus interpretaciones doctrinales; y la primera tarea que deberían 
abordar consiste en ponerse de acuerdo entre ellos mismos, lo que hasta la fecha no han logrado hacer.

Por último, Vine (1978), sin comprometerse totalmente en cualquiera de las tesis expuestas, plantea que la Psicología de la Religión es sin duda alguna un tema delicado, ya que su argumento está intimamente ligado a los sistemas básicos de valores de una persona; o lo que es igual, la Psicología de la Religión «sufre el riesgo del prejuicio».

Ringel (1978) también mantiene una tesis ecléctica; tiende hacia la colaboración multidisciplinar, eliminando prejuicios mutuos y abriendo nuevos grupos de trabajo entre psicólogos y teólogos.

Como colofón creemos conveniente mencionar las conclusiones a que se llegó en el último Congreso de Psicología de la Religión celebrado en Lancaster (1977), donde uno de los más persistentes presupuestos que alli se barajaban era el postulado por O'Doherty (1976), en el que se apoya la opinión de que sólo los "psicólogos religiosos» pueden comprender la religión lo suficientemente bien como para estudiarla justamente, y que los demás (ateos y agnósticos) deben atenerse a la búsqueda de correlaciones superficiales entre medidas de religiosidad y otras variables externas; en otras palabras, la Psicología de la Religión no puede ser estudiada por aquellos que adoptan una actividad agnóstica sobre el contenido de las creencias religiosas.

\section{LAS DIMENSIONES DE LA RELIGIOSIDAD}

Un tema en el que la mayoría de los estudiosos de la Psicosociología de la Religión están de acuerdo, hace referencia a la multidimensionalidad de las actitudes religiosas; hemos encontrado muy pocos trabajos que afirmen y etiqueten a la actitud religiosa como variable unidimensional.

Pero antes de matizar qué se entiende por multidimensionalidad en el área de las actitudes religiosas, deseariamos pergeñar algunas líneas acerca de los cuestionarios elaborados para evaluar la religiosidad. Las escalas al uso, arrastran, a nuestro juicio, ciertas lacras que hacen dificil una evaluación genuinamente psicosocial de la actitud religiosa. En primer lugar, son pruebas cuyos items contemplan posiciones «deístas» con respecto al hecho religioso (tal es el caso del "Cuestionario de Actitud Religiosa para Adolescentes de 12 a 18 años», de Moraleda [1977]). Por otro lado, dichos cuestionarios, o bien adolecen de la objetividad y tipificación necesarias, lo que a su vez dificulta, en cierto modo, su interpretación final (por ejemplo, el "Test I.P.A.R.A.»: Imágenes Proyectivas para el análisis de la Actitud Religiosa de los Adolescentes, de Moraleda [1976]), o bien obvian la consistencia de sus items, como en la «Religious Attitude Scale» de Francis (1972). Por último, recalcar que las escalas no evalúan una actitud positiva o ne- 
gativa frente al hecho religioso, sino que miden la «religiosidad» de los sujetos religiosos.

El término «multidimensionalidad» dentro del ámbito de las actitudes religiosas, no quiere sino enfatizar que la religiosidad «varía mucho en profundidad, amplitud, contenido y modo de funcionamiento» (Allport, 1957-1965-1968); o lo que es lo mismo, existen muchos modos de ser religioso. De ello se deriva que el concepto de religiosidad connota una serie de dimensiones o factores que han sido puestos de relieve en las investigaciones que a continuación exponemos.

Uno de los primeros estudios llevados a cabo en este sentido fue el de Allport (1954-1967), quien durante más de trece años estuvo distinguiendo entre una «religiosidad intrínseca» y otra a la que denominó «religiosidad extrínseca» o utilitaria. Estimó que los individuos extrínsecamente religiosos eran aquellos que consideraban la religión única o prioritariamente como un instrumento útil para la consecución de ciertos derechos y privilegios o para la defensa de otros ya alcanzados, es decir, como un medio para la obtención de otras cosas. Por el contrario, los intrínsecamente religiosos consideraban la religión como un fin en sí mismo, al cual debe ser subordinado todo lo demás. Dentro del tipo extrínseco, Allport distingue además dos dimensiones distintas: una, donde la religión tiende a vivirse como medio de poder económico y social y otra en la que se considera como norma y consuelo, guía, alivio y protección. De este análisis empírico que hace Allport, parecen vislumbrarse los tres planos de los que a otro nivel hablaba Kierkegaard: la religiosidad estética (de valores sensoriales, predominantemente económicos y sociales), una religiosidad ética (cuyos valores eran normativos y morales primordialmente) y una religiosidad religiosa (de valores intrinsecamente religiosos). En resumen, Allport apunta ya hacia la complejidad del fenómeno religioso, poniendo en entredicho los estudios que apoyaban la idea de la religiosidad como variable unidimensional.

Las aportaciones de Allport se vieron posteriormente secundadas por los estudios de Amón y Yela (1968) y Amón (1973), quienes analizando las dimensiones de la religiosidad concluyen que el continuo intrínseco-extrinseco no es unidimensional sino multidimensional.

Otro de los investigadores que trabajó e interesó en el campo de las actitudes religiosas fue Broen (1957), quien después de revisar los trabajos analíticos acerca de los factores que componen las actitudes religiosas, subrayó la idea de que era probable que tales aportaciones no fuesen totalmente fructíferas debido a la falta de conocimientos sobre cómo deberian ser analizadas las mismas. Se inclinaba hacia la suposición de que las actitudes religiosas estarian formadas por dos factores; un factor unipolar al que denominó "Cercanía a Dios» y en el cual las personas con saturaciones altas tenderian a sentir a Dios como muy real, cerca y accesible; tales sujetos, dice Broen, sienten que conversan con Dios, caminan y hablan con $\mathrm{El}$; su religiosidad consiste en 


\section{2}

recalcar la presencia amorosa y la guia de la Deidad en lugar de su función de juzgar. El segundo factor de Broen era bipolar y le asignó el nombre de "Fundamentalismo-Humanismow; las personas con cargas positivas en el polo "Fundamentalismo» tenderían a suponer que el hombre es esencialmente pecador; los individuos ubicados en el otro extremo del factor («Humanismo») aprecian que el ser humano posee el potencial necesario para hacer el bien, así como las herramientas para poner en marcha dicho potencial, desestimando con ello la necesidad de cualquier intervención divina. Así pues, los estudios de Broen, además de verificar la labor que Allport adujo años antes acerca de la existencia de diferentes e independientes factores en el campo de las actitudes religiosas, implican la necesidad de obtener medidas distintas en cada uno de los factores, con lo cual los resultados serían más satisfactorios a la hora de correlacionar dichas medidas con otras variables.

Con posterioridad a los trabajos expuestos, se sucedieron otros similares que corroboraron de nuevo la multidimensionalidad de las actitudes religiosas; David \& Revelle (1974) informan en sus investigaciones de la construcción de un "Inventario Multidimensional de Actitudes Religiosas", en el que distinguen cuatro escalas: la primera, bajo el título de «Profética», puntualiza un interés religioso por la justicia social, aunque no separa lo sagrado de lo secular; la segunda, a la que denominó "Sacerdotal", refleja una marcada distinción entre lo sagrado y lo secular y pone de relieve lo que hace referencia a la conducta ritual religiosa; a las dos escalas restantes les asignó el nombre de «Humanistico» y «Extrínseco», el contenido de las cuales correría parejo a los ya descritos por Broen de "Fundamentalismo-Humanismo".

En conexión con los estudios de David \& Revelle y reforzando sus conclusiones a favor de la multidimensionalidad de las actitudes religiosas, Law (1977) elaboró un «Inventario Multidimensional de Creencias Cristianas», donde aísla cuatro factores: al primero le dio el título de "Creencia General Cristiana" que evaluaba la religiosidad o "consentimiento del Credo" según Law, y que se podía equiparar al factor de «Creencia en Dios» descrito por Broen; el segundo factor, «Creencia Católico-Romana», proporcionaba diferencias generales entre las creencias protestantes y católicas; el tercer factor fue denominado "Confianza en el hombre», y reflejaba la seguridad de un sujeto en la integridad y racionalidad del hombre, así como en la habilidad del mismo para dar forma a su destino; por último, el factor titulado «Dios colérico y Creencias moralistas» expresaba una visión puritana de la vida y la creencia en un Dios severo y vengativo. Los dos últimos factores que Law extrajo se asemejan a la dimensión bipolar de «Fundamentalismo-Humanismo» descrita por Broen.

Como hemos tenido la ocasión de comprobar a lo largo de la descripción de los estudios que se han realizado en materia de actitudes religiosas, el contenido de los factores obtenidos en una investigación 
es muy similar al contenido de las dimensiones que ofrecen otros estudios al respecto. Ello parece una prueba lo suficientemente consistente como para afirmar la validez de la perspectiva multidimensional.

Aparte de los estudios citados, se han llevado a cabo otros, cuyas conclusiones han corroborado la existencia de factores independientes, al menos dentro de la tradición cristiana, como los de Fukuyama (1961), Putney y Middleton (1961), Lenski (1963), King (1967), King y Hunt (1975). Evidencian que lo religioso psicológicamente considerado no viene vertebrado por una sola dimensión simple y única sino factorialmente compleja.

\section{PANORAMA DE LAS INVESTIGACIONES EN PSICOLOGIA DE LA RELIGION}

Vamos a hacer una apretada revisión de trabajos que se han ocupado de la psicosociología de la religión, aclarando que son pocos los de contenido teórico que formulan modelos comprensivos o delimitaciones epistemológicas, antes bien, se trata de estudios descriptivos y correlacionales, de conclusiones problemáticamente generalizables.

Las investigaciones más usuales se ocupan de correlacionar la actitud religiosa, la creencia religiosa o la religiosidad con otras variables de personalidad entre las que cabe destacar: autoritarismo, neuroticismo-estabilidad, autoconcepto, autoestima y ansiedad. Para mayor claridad, parece conveniente hacer el comentario por bloques; empezaremos por los referidos al autoritarismo.

Uno de estos estudios ha sido el de Martin y Nichols (1962); hasta esa fecha existian aportaciones clínicamente orientadas, confirmando que la persona religiosa se mostraba más conformista y defensiva, mientras que la no religiosa era más independiente. Por aquel entonces, había también pruebas de que la creencia religiosa correlacionaba positivamente con la escala California «F» de autoritarismo y negativamente con inteligencia. Por si fuera poco, Khanna (1957) describió la personalidad religiosa como más autoritaria, etnocéntrica y perceptual e intelectualmente más rígida que la persona no religiosa. Estimulados por conclusiones tan negativas, Martin y Nichols se proponen renovar dicha imagen. Para ello, administran escalas de creencias e información religiosa, el PA, L y MP del MMPI, la escala California «F» de autoritarismo y un cuestionario de ambiente social a 59 varones y 104 mujeres estudiantes no graduados. Los resultados obtenidos muestran el fracaso en verificar descubrimientos previos respecto a las correlaciones de personalidad autoritaria y creencias religiosas. Por el contrario, señalan una correlación de (.18) entre creencia religiosa y autoritarismo, arguyendo que las correlaciones de personalidad y creencia religiosa encontradas en otros estudios, son mucho más elevadas debido a factores casuales, aunque Martin y Nichols no especifican con nitidez la naturaleza de los mismos. 
A estos trabajos sucedieron los de Cline y Richards (1965). Estudiaron los factores que componen la creencia y la conducta religiosa, investigando así los índices de religiosidad mediando la variable sexo. Las pruebas que utilizaron fueron las siguientes: un test proyectivo tipo T.A.T., una entrevista intensiva y profunda y un cuestionario Likert de 67 items de creencia y conducta religiosa. El objetivo prioritario residia en aclarar algunos estereotipos sostenidos en anteriores trabajos tales como ulas personas religiosas son más autoritarias que las no religiosas». Sus resultados concluyeron que un no creyente es capaz de ser tan autoritario sobre sus propias creencias como un creyente con respecto a sus dogmas de fe. Al mismo tiempo apunta, que es preciso tener presentes las diferencias sexuales en el análisis de las creencias religiosas, lo que recientemente ha recabado en sus investigaciones Francis (1981). En otro estudio (Cline y Richards, 1965), se mostró que los individuos que computaban altas medidas de religiosidad en las «Escalas de Opinión Pública de la Universidad de California», tendían a ser más rígidos, dogmáticos, inflexibles y conformistas que los sujetos cuyas puntuaciones en religiosidad eran menos elevadas.

Otros investigadores han intentado demostrar que existe una correlación positiva entre el compromiso religioso y una personalidad relativamente defensiva y autoritaria (Ranck, 1961; Stanley, 1964). En más recientes investigaciones (Rohrbaugh y Jessor, 1975) se reafirma la caracterización del individuo religioso como convencional y conformista con las instituciones sociales. Por último, Heintzelman y Fehr (1976), correlacionando la modificación de Brown del "Test de Ortodoxia Religiosa» de Thouless con la «Escala de Ansiedad Manifiesta» de Catell, en un grupo de 82 estudiantes de primer curso de Psicología, afirman que existe una correlación negativa significativa entre las variables mencionadas.

Veamos a continuación los estudios sobre neuroticismo-estabilidad y ansiedad.

Ante todo, es preciso adelantar que los resultados obtenidos son tan poco definitivos como los hallados en los estudios sobre autoritarismo y religiosidad. Las primeras investigaciones fueron llevadas a cabo por Wilson y Miller (1968) al administrar a 100 estudiantes no graduados de la Universidad de Alabama un formulario-extracto de la «Escala de Ansiedad Manifiesta» de Taylor, junto con una "Prueba de aprensión» que contenia expresiones de miedo a estímulos comúnmente temidos tales como enfermedad, animales, fuego, accidentes, etc... y un cuestionario donde los sujetos se autoevaluaban con respecto a diversas prácticas religiosas: asistencia a la Iglesia, creencia en un ser supremo, inmortalidad del alma, moralidad religiosa, etc. Los resultados evidenciaron correlaciones positivas significativas entre religiosidad y miedo (.92) y religiosidad y ansiedad (.81). Con este trabajo, Wilson y Miller apuntaron la existencia de una pequeña pero clara tendencia 
de las personas no religiosas a dar respuestas más equilibradas en lo que concierne a la ansiedad y miedo.

Años más tarde, las investigaciones de Heintzelman y Fehr (1976) cuestionaron las conclusiones anteriormente citadas, al correlacionar los puntajes obtenidos en el "Indice de Religiosidad» de Thouless y la «Escala de Ansiedad Manifiesta» de Catell, lo que arrojó un coeficiente no significativo; esta conclusión estaba de acuerdo con una investigación previa (Brown, 1962) que no detectó ninguna correlación significativa entre religiosidad y ansiedad manifiesta. Fehr y Heintzelman (1977) replicaron sus estudios para verificar los trabajos publicados por ellos mismos años antes. Así, administraron a 120 estudiantes de primer curso de Psicología el "Test de Ortodoxia Religiosa» de Thouless y la "Encuesta de valores" de Allport-Lindzey-Vernon para obtener dos medidas de religiosidad y correlacionarlas con la «Escala de Ansiedad Manifiesta». Los resultados tampoco detectaron correlaciones significativas.

Siguiendo con los estudios sobre religiosidad y ansiedad, McClain (1978), analizó las diferencias de personalidad en base a los puntajes extraidos en EPPS, CPI y 16 PF, en un grupo de 145 sujetos religiosos y otro de 133 no religiosos detectados por medio del «Inventario de Orientación Religiosa» de Allport; los resultados indican que los individuos intrinsecamente religiosos son a la vez más autocontrolados y mantienen una mejor adecuación personal y social que los no religiosos; estos últimos, dice McClain, son más «inquietos».

Sturgeon y Hamley (1979), apoyan las conclusiones de McClain. Administraron una "Escala de Ansiedad Existencial», un "Inventario de Ansiedad» de Spielberg, la «Escala de Orientación Religiosa» y la «Escala de Localización interna-externa del control» de Rotter a 144 estudiantes vinculados a una iglesia protestante conservadora. En resumen, los sujetos intrínsecamente religiosos aparecían significativamente menos ansiosos existencialmente, mostrando mayor «locus interno» del control que el grupo extrínsecamente religioso.

La investigación más reciente que hace referencia a la relación del neuroticismo con la religiosidad se debe a Francis et al. (1981); obtuvieron puntuaciones de la «Escala de Neuroticismo» de Eysenck y la «Escala de Actitudes Religiosas» de Francis, de 1.088 sujetos de ambos sexos, estudiantes ingleses, comprendidos entre las edades de $15 \mathrm{y}$ 16 años. Sus conclusiones confirman una correlación positiva y significativa entre religiosidad y neuroticismo, aunque cuando Francis interprete sus datos añadirá que dicha correlación ues un artificio de las diferencias sexuales» de los sujetos que componen la muestra del estudio, ya que las mujeres obtienen rasgos significativamente mayores que los varones, tanto en la escala de neuroticismo como en la de religiosidad. Al finalizar su trabajo, Francis preferirá no apoyar la hipótesis de que la religiosidad es una expresión más de la inestabilidad, ni tampoco que la religiosidad sustenta la estabilidad. 
Por consiguiente, los datos obtenidos en este sentido, al correlacionar las variables de neuroticismo y ansiedad con las medidas de religiosidad no son de manera alguna congruentes. Asi Fehr y Heintzelman no hallan ninguna relación significativa en estas direcciones; McClain, Sturgeon \& Hamley y Brown sugieren que la religiosidad está asociada con un mayor y mejor ajuste social y estabilidad personal; Wilson y Miller por el contrario apoyan la idea de que la religiosidad correlaciona con baja autoestima, mayor ansiedad y alto neuroticismo.

Mencionaremos ahora los trabajos publicados sobre religiosidad y autoestima. Tales investigaciones al igual que las anteriores arrojan conclusiones incoherentes, además de escasas. Los primeros estudios sobre religiosidad y autoestima se atribuyen a Cowen (1954) y Strunk (1958). Cowen obtiene correlaciones negativas entre religiosidad ortodoxa y los cómputos de autoestima. En el trabajo de Strunk se escogieron a 136 alumnos, 55 varones y 81 mujeres, los cuales rellenaron el «Inventario de Autoevaluación» de Brownfair (únicamente el formulario de carácter privado) y un "Indice de Religiosidad» construido específicamente al efecto; los resultados confirmaron que los sujetos con un autoinforme relativamente afirmativo tendian a obtener puntuaciones más elevadas en el cuestionario de religiosidad, que los autoinformes caracterizados menos afirmativamente. Para Strunk, esto prueba que la autorrealización en el dominio psicológico está conectada con la fe religiosa; es decir, un adolescente que cree en Dios como ayuda presente, no sólo cuando tiene problemas, sino también como una fuente diaria de guía para su mejor desarrollo, tiene un cierto recurso de fuerza y coraje ante dificultades cotidianas.

Los resultados de Strunk parecían de este modo contradecir las tesis planteadas por Cowen. Sin embargo, ahondando sobre el asunto, Heintzelman y Fehr (1976) probaron que las correlaciones encontradas al administrar el «Test de Ortodoxia Religiosa» de Thouless y el «Inventario de Autoestima» de Coopersmith no eran significativas. Estos autores intentarán un año después corroborar los datos que habían obtenido; con tal propósito, extraen medidas de religiosidad con el "Test de Ortodoxia Religiosa» de Thouless, que ya utilizaron en sus investigaciones precedentes, y de la «Encuesta de valores» de AllportLindzey-Vernon; los puntajes son correlacionados con medidas de autoestima, llegando a la conclusión de que parece existir una correlación mínima negativa entre ambas medidas, lo que venia a apoyar de nuevo las teorias apuntadas por Cowen dos décadas antes.

Como hemos podido comprobar, los estudios se limitan normalmente a la interpretación de correlaciones entre las variables de religiosidad o creencia religiosa con otras que pueden reducirse a tres: neuroticismo, autoritarismo y autoconcepto; las coordenadas, pues, por las que transcurren en estos momentos las investigaciones sobre el tema tienen un tono descriptivo-correlacional, con evidente ausencia de marcos teóricos abarcadores y explicativos. 


\section{Estudios}

\section{Resumen}

El objetivo del trabajo radica en llevar a cabo una revision sobre el estado actual de las investigaciones referentes a los aspectos psicosociales de la religion.

Se comienza planteando los problemas que ello extraña: escasez de investigaciones rigurosas, connotaciones ideológicas de los planteamientos y estrategias, diversas y contrapuestas acepciones de términos elementales, etc.

Se aborda la polémica que ba enfrentado a psicólogos y teólogos, que, a pesar de su poca operatividad, ba supuesto un foco de interés.

Se analiza la multidimensionalidad de las actitudes religiosas, aportaciones realizadas al respecto y los instrumentos de medida que ban gozado de mayor predicamento.

Se concluye con la exposición panorámica de las investigaciones actuales más relevantes sobre el tema, señalando cómo se ban ocupado básicamente de correlacionar las actitudes religiosas o la religiosidad con diversas variables de personalidad, como autoritarismo, neuroticismo, autoestima, etc.

\section{Summary} gion.

The purpose of this paper is to revise the current research on psychosociological aspects of reli-

We begin by pointing out the problems witch this type of work entails: scarcity of rigurous research, ideologically connoted strategies, and contradictory interpretations of basic terms, etc.

Then we deal with the debate with has set psychologist and theologicians on opposite sider, and witb, in spite of its low operativeness it has meant a focus of interest.

The multidimensionality of religious attitudes, new contribution in the field, and the mechanism of assessment which bave enjoyed higher prestige are also analized bere.

The paper concludes with a panorama of the more relevant research proyect on the subject, and it emphatizes the fact that bave been concerned with correlations religious attitudes with the diffe-. rent variables of personality such as autboritarianism, neuroticism and self-esteem.

\section{Referencias}

Allport, G.: The nature of prejudice. Addison-Wesley; Cambridge Mass, 1954.

ALLPORT, G.: The individual and bis religion. Macmillan, New York, 1957.

AllPORT, G.: «Behavioral Science, religion and mental health». Jr. of Religion and Health, vol. 2, n." 3, $1963,187-197$.

AlLPORT, G.: "The force of racial differences in human relations. Prejudice: is it societal o personal?» Religious Education, January-February, 1964, 20-29.

ALLPORT, G.: Patiern and growth in personality. Holt, Rinehart \& Winston. New York, 1965.

ALLPORT, G.: "The religious context of prejudice». Tbe Graduate Journal, VII, Supplement, 1966, $115-130$.

Allport, G., y Ross, J.: "Personal religious orientation and prejudice». Jr. of Person. and Soc. Psycbol., n. 4, $1967,5,432-443$.

AllPORT, G.: The person in psycbology. Beacon Press. Boston, 1968.

AMÓN, J., y YelA, M.: «Dimensiones de la religiosidad». R. P. G. A., vol. XXIII, n. ${ }^{\circ} 95,1968,989-993$.

AMÓN, J.: "Nuevo análisis sobre la dimensionalidad de la religiosidad utilitaria». R. P. G. A., vol. 28 , $1973,122,319-332$.

Argyle, M. \& Beit-Hallahmi, B.: Tbe Social Psycbology of Religion. Rotledge \& Kegan Paul. London, 1975.

BAGLEY, G.; BOSHIER, R., y NIAS, D.: «The ortogonality of religious and racialist-punitive attitudes in three societies». The Jr. of Soc. Psycbol., 1974, 92, 173-179.

BRoEN, W.: «A factor analytic study of religious attitudes». Jr. of Abmormal and Soc. Psychol., 1957, 54, 176179.

Brown, L.: «A study of religious belief». British Jr. of Psychol. 1962, 53, 259-272.

Clayton, R., y Gladdin, J.: «The five dimensions of religiosity». Jr. Sci. Study of Religion, 1974, 13, 135143.

Cline, V., y Richards, J.: «A factor analytic study of religious belief and behaviom. Jr. of Person. and Soc. Psychol., 1965, 1, 569-578.

COWEN, E.: «The negative concept as a personality measure». Jr. Consult Psychol., 1954, 18, 138-142.

DAviD, B., y REVELLE, W.: «A multidimensional religious attitude inventory related to multiple measures of race». The Jr. of Soc. Psycbol., 1974, 92, F. H. 
Diaz Murugarren, J.: "La Iglesia como neurosis obsesiva». Rev. de Estudios Filosóficos, 1977, 26, 463-511.

Digenan, S., et al.: «Religious beliefs, religious commitment and prejudice». Jr. of Soc. Psycbol., 1975, 97, 147-148.

DREGER, R.: "Some personality correlates of religious attitude as determined by proyective techniques». Psychological Monographs, 1952, 16, 3.

FehR, L., y HeINTZFLMAN, M.: "Personality and attitudes correlates of religiosity: a source of controversy». The Jr. of Psychol. 1977, 95, 63-66.

Francis, L.: "Religious Attitude Scale». Departament of Education. University of Cambridge. Cambridge, 1972.

FranciS, L.: "The Psychology of Religion: revived, not yet reborn». Bull. Br. Psycbol. Soc., 1978, 31, 4445.

FrancIS, L., et al.: "The relationship between neuroticism and religiosity among english 15 and 16 yearolds"). Jr. of Soc. Psychol., 1981, 114, 99-104.

FukUyamA, Y.: "The mayor dimensions of church membership". Rev. of Religious Research, 1961, 2, 15461.

Grigory. W.: «The ortodoxy of the authoritarian personality». Jr. of Soc. Psychol., 1957, 45, $217-232$.

Harris, D.: "Correspondence". Bull. Br. Psychol. Soc., 1978, 31, 130-131.

HEINTZFLMAN, M., y FEHR, L.: «Relationship between religious ortodoxy and three personality variablesn. Psychol. Reports, 1976, 38, 756-758.

KHANNA, J.: "A study of the relationship between some aspects of personality and certain aspects of religion beliefs). Dissertation Abstracts, 1957, 17, 2696-2697.

KING, M.: "Measuring the religious variable: nine proposed dimensions». Jr. for the Sci. Study of Religion, 1967, 6, 173-190.

KING, M., y HuNT, R.: «Measuring the religious variable: national replication». Jr. for The Sci. Study of Religion, 1975, 14, 13-22.

LAW, G.: "A multidimensional inventory of Christian beliefs". Jr. of Soc. Psycbol., 1977, 102-325.

LENSKI, G.: The religious factor: A sociological study of religion's impact on politics, economics and family. Doubleday. Garden City, N. Y., 1961.

LENSKI, G.: The religious factor. Doubleday. Garden City, N. Y., 1963.

LFVINE, C.: "Role-Taking standpoint and adolescence usage of Kolberg's conventional stages of moral reasoning". Jr. of Person. and Soc. Psychol., n. ${ }^{\circ} 1,1976,34,41-46$.

Mankeliunas, M.: Psicologia de la Religiosidad. Religión y Cultura. Madrid, 1961.

Martin, C., y Nichols, R.: «Personality and religion beliefs». Jr. of Soc. Psychol., 1962, 56, 3-8.

MCCLAIN, E.: "Personality differences between intrinsically religious and nonreligious students: a factor analytic study». Jr. of Person. Assesm., 1978, 42-2.

MCDOWELL, M.: Tecbniques used in attitudinal measurement in religion: 1950-60. Salt Lake City, Utah; Westminster College, 1963.

Michates, J.: A survey of the empirical psychological study of religion of the past twenty-five years. Mimeo. Los Angeles, 1956.

MORAlEDA, M.: «Test I.P.A.R.A.: Imágenes Proyectivas para el Análisis de la Actitud Religiosa de los Adolescentes». Instituto Superior de Ciencias Catequéticas. Ed. San Pio X. Salamanca, 1976.

Moraleda, M.: "Cuestionario de Actitud Religiosa para Adolescentes de 12 a 18 años". Instituto Superior de Ciencias Catequéticas. Ed. San Pio X. Salamanca, 1977.

Moraleda, M.: La actitud religiosa de los adolescentes. Ed. Bruño. Madrid, 1977.

O'DOHERTY, E.: "Grace, faith and the human person". Lancaster Colloguium on the Psychology of Religion. Lancaster, 1976.

Poveda, J.: “Psicologia de la Religión». Revista de Espiritualidad. 1967, 26, 187-198.

Putney, S., y MidDLETON, R.: «Dimensions and correlates of religious ideologies». Social Forces, 1961, 39, 285-290.

RANCK, J.: «Religious conservatism-liberalism and mental health». Pastoral Psychology, 1961, 12, 34-40.

RINGEL, E.: «Religion und neurose». Herkon, 1978, 32, 174-182.

ROHRBAUGH, J., y JESSOR, R.: «Religiosity in youth: a personal control against deviate behavior». Jr. of Person., 1975, 43, 110-124.

SChwartz, M., et al.: "Duration of effect of Good Mood on helping: Foot-prints on the stands of time». Jr. of Person. and Soc. Psychol., 1976, 34, n..$^{\circ}$, 385-393.

Stanley, G.: "Personality and attitude correlates of religious conversion». Jr. for the Sci. Study of Religion, $1964,4,60-63$.

Stollberg, D.: «Psicología de la religión». En Arnold-EySENCK-Meill: Diccionario de Psicologia. Ed. Rioduero. Madrid, 1979.

STRUNK, O.: «Relation between self-report and adolescent religiosity». Psycbol. Reports, 1958, 4, 683-686.

Sturgeon, R., y Hamley, R.: "Religiosity and anxiety». Jr. of Soc. Psychol., 1979, 108, F. H.

TORNOS, A.: «Un punto de vista psicológico sobre religiosidad popular». Rev. Fomento Social, 1970.

Vergote A.: Psicologia Religiosa. Ed. Taurus. Madrid, 1969.

VINE, I.: «Facts and values in the Psychology of Religion». Bull. Br. Psychol. Soc., 1978, 31, 414-417.

WILSON, W., y Miller, H.: «Fear, anxiety and religiousness». Jr. Sci. Study of Religion, 1968, 7, 111. 